\title{
TEXTO DIGITAL E INTERNET: HACIA UNA NUEVA LEGITIMACIÓN Y USO DE LA BIBLIOGRAFÍA CIENTÍFICA EN EDUCACIÓN MUSICAL
}

\author{
Pablo Ramos Ramos
}

IES Carrús de Elche (Alicante)

pablo.ramos.ramos@gmail.com

Este trabajo de síntesis estudia el extraordinario incremento de bibliografía científica en educación musical, resultado de la aparición de las últimas tecnologías de la información y la comunicación. Se analizan también las nuevas herramientas bibliográficas necesarias para manejar dicho exceso informacional, sin las cuales el proceso científico podría verse retrasado y alterado.

This review has been carried out in order to show the consequences of the extraordinary growth in the scientific literature in music education as a result of the use of the latest information technologies. New bibliographic tools to handle the excess of information, without which the scientific process could be distorted and delayed, are discussed.

\section{EL AUMENTO DE LA BIBLIOGRAFÍA CIENTÍFICA EN EDUCACIÓN MUSICAL}

Las nuevas tecnologías digitales y de redes están induciendo cambios profundos en la generación, transmisión y apropiación de la información. Este proceso no sólo nos afecta de una forma externa sino que va más allá: la neurociencia viene demostrando que el trabajo continuo con Internet y con grandes cantidades de textos hace que leamos y procesemos el contenido de una manera diferente a como lo hacíamos anteriormente (SMALl y VORGAN, 2009). Aun así, la saturación informativa es tal que hemos necesitado desarrollar nuevas herramientas para gestionarla. Esta realidad ha llevado al mundo de la investigación científica a transformar sus metodologías -sobre todo en lo que al tratamiento de fuentes se refiere.

A lo largo del siglo XX, las ciencias experimentales, así como las ciencias sociales, fueron incrementando gradualmente su producción de textos -revistas, libros, manuales- en un proceso que eclosionaría con la llegada de los formatos digitales. En el caso de la investigación en educación musical, la creación de un corpus sólido de conocimientos científicos tardaría algo más en llegar. Entre las décadas de 1950 y 1980 comenzaron a aparecer las obras que sentarían las bases de la futura investigación en nuestra área: La perception de la musique de Robert FRANCÈS (1958), L'acquisition des structures tonales chez l'enfant de Michel IMBERTY (1969) y The musical mind de John SLOBODA (1985). Asimismo, la creación de revistas especializadas potenció este florecimiento: Journal of Research in Music Education (fundada en 1953), Psychology of Music (1973), Music Perception (1980), International Journal of Music Education (1983). España, por su parte, intentaría seguir la estela internacional con la creación de revistas como Música y Educación (1988) o Eufonía (1995).

Gracias a la generalización de internet y del documento electrónico, este fértil panorama se ha visto sobrepasado, no sólo por la mayor facilidad para editar textos sino, además, por la posibilidad de tener acceso a publicaciones de todos los rincones del mundo. El rápido incremento de la bibliografía en educación musical se encuentra claramente reflejado en los datos del Répertoire International de Littérature Musicale (RILM), pasando de cerca de 10.000 entradas en el año 1987 hasta casi 40.000 en 2007.

Esta evolución ha supuesto para la investigación en nuestro campo una ruptura mucho más pronunciada que en el caso de otras ciencias con más tradición editorial; lo cual supone un problema, ya que, dada la "abordable" cantidad de información que poseíamos antes de la llegada de Internet, los investigadores en educación musical no hemos desarrollado herramientas eficientes de selección y uso de textos. Por ello, la mayor dificultad que encontramos hoy es la discriminación cualitativa de nuestra bibliografía científica. Para entender esta problemática es necesario analizar la forma en que trabajamos con la Red y el tratamiento que hacemos de las fuentes en nuestras investigaciones. 


\section{LA REVISTA DIGITAL Y LA NORMALIZACIÓN DEL DOCUMENTO ELECTRÓNICO EN INVESTIGACIÓN}

La comunidad científica afronta esta eclosión documental con ilusión y, al mismo tiempo, con recelo. Uno de los principales motivos de esta desconfianza está relacionado con el exceso de información; a saber: el trabajo prolongado con hipertextos -que conducen a una infinidad de fuentes- produce una "desorientación cognitiva" (BACCINO, 2004: 55), lo cual, trasladado al mundo académico, puede llevar al retraso y la alteración de la tarea investigadora. Este problema se ve agravado por la multiplicidad de formatos digitales existentes - generando dudas en torno a la legitimidad, integridad y citación de los textos. Así pues, el investigador encuentra problemas a la hora de identificar, localizar y manejar los documentos más idóneos para su trabajo. En este sentido, la estandarización del material científico y de las técnicas de búsqueda supone un avance importante. Este proceso se ha concretado con la generalización de la revista electrónica como principal canal para la transmisión del conocimiento científico en Internet.

Una de las razones por las que se ha optado por el modelo de la revista electrónica, normalmente en formato pdf, es que permite tratar el documento como un texto impreso (personalmente, pensamos que la citación de documentos electrónicos mediante su dirección web y su fecha de consulta es una fuente constante de problemas, ya que la URL puede cambiar en cualquier momento). Este proceso es posible gracias al digital object identifier (DOI), número de control creado para identificar los “objetos digitales" (revistas digitales o partes de ellas).

Por otro lado, hay que destacar que la proliferación de revistas científicas online se ha producido, en parte, gracias a la aparición de programas de software libre como el Open Journal Systems, que permite crear y gestionar todo el proceso editorial de una revista digital. En España, por ejemplo, este programa es aplicado por el CSIC y por el consorcio de Revistas Catalanas con Acceso Abierto (RACO).

Ahora bien, aunque la revista electrónica parece ser la solución a la heterogeneidad de los formatos digitales, creemos necesario desarrollar dos reflexiones importantes en cuanto a su futuro: una relativa a su relación con las ediciones en papel y otra sobre la gratuidad del acceso a sus contenidos.

\section{Digital contra impreso: ¿el fin de una era?}

Actualmente, hay reticencias a abandonar el formato impreso por razones de calidad, existiendo un duro debate en el seno de la comunidad científica sobre la conveniencia de editar o no directamente en digital. Si bien la revista electrónica permite una mayor difusión -siendo éste uno de los objetivos principales de tales publicaciones-, la edición en papel, al tener un proceso más complejo de elaboración, goza de un mayor prestigio entre los expertos. Desde nuestro punto de vista, tal controversia no tiene fundamento ya que ambos formatos, papel y electrónico, pueden coexistir sin problemas o, más aún, complementarse.

Según un estudio desarrollado en el Instituto de Historia de la Ciencia y Documentación López Piñero de la Universidad de Valencia-CSIC (OSCA LLUCH et al., 2008:153) el papel es todavía el soporte mayoritario para revistas científicas, con un $60 \%$ del total, mientras que la disponibilidad combinada en papel y electrónica está ya en un 31\% (sobre todo por la digitalización de ediciones completas) y sólo electrónica en un $9 \%$, aunque en rápido crecimiento. De esta manera, es muy probable que la edición digital acabe imponiéndose a la impresa, la cual, como sugiere Vicente LiLlo (2009:8), responsable de Revistas de la Universidad de Murcia, podría quedar para "fines protocolarios o ediciones especiales". 


\section{El problema de la gratuidad}

Determinar si las revistas científicas digitales serán gratuitas en un futuro es algo arriesgado ya que, a día de hoy, no existe unanimidad al respecto. Muchos expertos, como GIMÉNEZ TOLEDO (2006:118), sugieren que la generalización del acceso libre y gratuito a los trabajos científicos por medio del texto electrónico es un proceso imparable. Sin embargo, ciertas instituciones de gran peso - principalmente americanas y británicas- siguen políticas de no gratuidad, más allá de los clásicos intercambios con revistas de otras universidades. La mercantilización del saber, tal y como es concebida en Estados Unidos, se concreta en grandes empresas que gestionan los recursos científicos en línea. Una de estas empresas es SAGE, editor independiente que aglutina a más de 500 revistas y bases de datos en humanidades, ciencias sociales y medicina. Sólo en nuestra área de estudio agrupa las siguientes revistas: International Journal of Music Education, Journal of Music Teacher Education, General Music Today, Journal of Research in Music Education, Music Educators Journal, Psychology of Music y Research Studies in Music Education.

Si bien muchas universidades españolas tienen contratados los servicios de SAGE, ofreciendo acceso gratuito e íntegro a los documentos, se puede comprobar la importancia del negocio generado al ver el precio por artículo para un subscriptor individual, 25 dólares, mediante el denominado "short-term access", que permite consultar el texto sólo durante 24 horas -sin posibilidad de descarga. Es por ello que, más que en la realidad anglófona, las afirmaciones de GiMÉNEZ TOLEDO (2006) se enmarcan en un pensamiento europeo de democratización del conocimiento.

A pesar de ello, están surgiendo iniciativas puntuales en el mundo anglosajón que persiguen el acceso gratuito a la información científica. Es el caso, por ejemplo, del British Journal of Music Education, editado por la Universidad de Cambridge (Cambrigde Journals) o del Research and Issues in Music Education, de la americana University of St. Thomas (en formato HTML).

Así pues, la pregunta que podemos hacernos es si el modelo gratuito puede competir -en lo que a calidad se refiere- con estas importantes revistas de pago. Para responderla adecuadamente podemos citar el Directory of Open Access Journals, que reúne revistas gratuitas de varios países, destacando una treintena de publicaciones sobre investigación musical. Este directorio es una muestra de la diversidad de propuestas serias y gratuitas que se pueden encontrar en Internet, como la publicación turca Journal of Interdisciplinary Music Studies, la danesa Journal of Music and Meaning o la alemana Zeitschrift für Kritische Musikpädagogik (debemos señalar que estas revistas están formadas por excelentes comités editoriales). En España, la Revista Electrónica Complutense de Investigación en Educación Musical -Universidad Complutense de Madrid- y la Revista Electrónica de LEEME (Lista Europea Electrónica de Música en la Educación) -Universidad de La Rioja- son buena muestra de ello. Otra publicación gratuita digna de mención es el Journal de Recherche en Éducation Musicale -Universidad de París IV, Sorbona-, ya que su comité científico cuenta con lo más granado de la investigación en educación musical de Francia. En esta misma línea se encuentra también la canadiense Recherche en Education Musicale, de la Universidad de Laval en Quebec.

Existe, además, una fórmula intermedia en lo que concierne a la gratuidad de las revistas: ofrecer un ejemplar para todos los miembros de la asociación que la edita. Como por ejemplo la Società Italiana per l'Educacione Musicale, que entrega su revista Musica Domani a todos sus socios o la International Society for Music Education (ISME) que ofrece los cuatro números anuales de su International Journal of Music Education a los miembros de la sociedad -para integrarla hay que pagar una cuota bianual.

Finalmente, debe destacarse que dentro de esta línea de difusión libre del conocimiento están surgiendo propuestas que no están ligadas a ninguna institución en particular y que, sin embargo, son de una excelente calidad. Tal es el caso de la revista Action, Criticism, and Theory for Music Education (surgida del colectivo Maydaygroup). 


\section{BÚSQUEDA Y RECUPERACIÓN DE LA INFORMACIÓN}

Como acabamos de ver, la llegada del texto electrónico y de internet ha favorecido un desarrollo exponencial de la bibliografía científica en educación musical. Se abre ante nosotros un nuevo universo de posibilidades que, sin embargo, puede transformarse en una pesadilla si no sabemos navegar por él.

Lo más complicado al iniciar una investigación ya no es buscar hipótesis apropiadas frente a una problemática, sino saber quién ha trabajado antes sobre ese tema y qué obstáculos ha encontrado. En este sentido, el campo de la bibliotecnología ha desarrollado complejas bases de datos y motores de búsqueda que permiten acotar los límites de una ciencia y su producción textual, por lo que la investigación del siglo XXI comienza en internet antes que en la biblioteca. Además, la principal novedad de estas herramientas es que, gracias al protocolo OAI-PMH (Open Archives Initiative Protocole for Metadata Harvesting), se puede recuperar la información solicitada, es decir, acceder al texto completo.

Ahora bien, surge un problema capital ligado a la indexación de los documentos, ya que cada base de datos desarrolla unos criterios propios de selección, lo que da como resultado que no todos los textos se puedan localizar en todos los motores de búsqueda. Así pues, las siguientes páginas no pretenden ser un compendio de bases de datos en las que encontrar toda la bibliografía científica en educación musical, sino una pequeña muestra de las posibilidades que se abren ante nosotros.

\section{Bases de datos en castellano}

En España, varios organismos públicos han realizado grandes esfuerzos en la creación de bases de datos bibliográficas. Una de las más importantes es la ISOC (Ciencias Sociales y Humanidades) que, producida por el Consejo Superior de Investigaciones Científicas, contiene la producción científica publicada en España desde la década de 1970, lo que la convierte en una de las principales herramientas de búsqueda en nuestra área. Muestra de ello es que, en una búsqueda realizada en octubre de 2010, el descriptor "educación musical" ofreció 1177 entradas, de las que, eso sí, sólo 108 enlazaron a texto completo -tal vez porque en nuestro país las revistas en papel siguen pesando más que las digitales. Cada entrada incluye información básica del documento como autores, ISSN, datos de la fuente y enlace con la revista editora (Fig. 1).

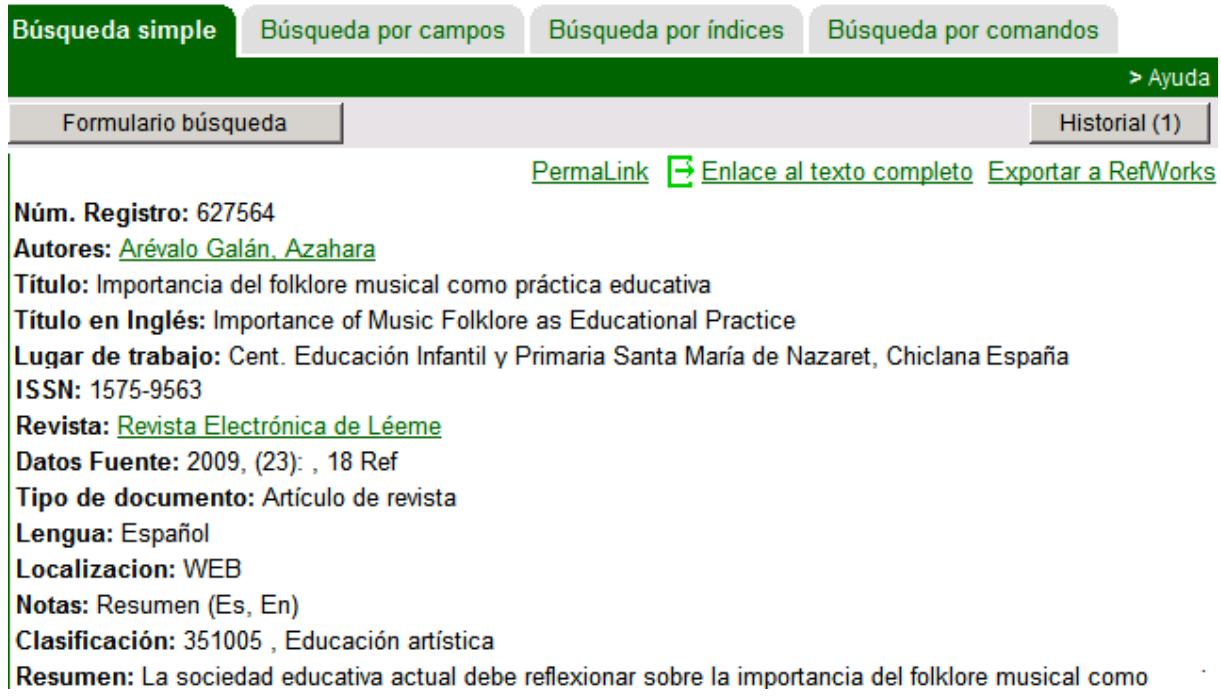

Fig. 1 
Otro punto a destacar es que la ISOC permite exportar la información a gestores bibliográficos (como RefWorks, EndNote o Zotero), posibilitando la creación de bases de datos personalizadas y etiquetas de las diferentes entradas mediante enlaces permanentes (Permalink) para una mejor localización en el futuro.

Existen además otras bases de datos de gran importancia. Redined, desarrollada por el Ministerio de Educación, se centra en investigación educativa y destaca por la gran cantidad de documentos indexados. También son de enorme utilidad: Compludoc, creada por la Universidad Complutense de Madrid; Dialnet, de la Universidad de La Rioja; y Teseo, del Ministerio de Educación, que recoge la información sobre las tesis doctorales presentadas en España desde 1976. Además, en el ámbito iberoamericano en general debemos destacar la gran aportación de Latindex, que reúne tres grandes bases de datos con información científica de América Latina, el Caribe, España y Portugal.

\section{Las grandes empresas de gestión de la información}

Si bien en España este gran avance en materia de bibliotecnología ha sido posible gracias a instituciones públicas, en el área anglosajona son grandes empresas privadas las que están a la cabeza de la innovación documental. Entre las cuatro más importantes se encuentran Proquest, Ebsco, Scopus y Thomson Reuters (que ha creado la Web of Knowledge). A través de sus portales podemos acceder a lo más relevante de la información científica en nuestra área a nivel mundial. Un ejemplo de ello es que el acceso al Répertoire International de Littérature Musicale (RILM) sólo se realiza a través de Proquest y Ebsco, además de OCLC. Otro ejemplo: los artículos de las revistas de pago más destacadas en educación musical -como Research Studies in Music Education e International Journal of Music Education- son distribuidos mediante subscripción a estas compañías. Ahora bien, el acceso a nivel individual no es viable por su elevado coste, siendo universidades e instituciones científicas sus principales clientes (al ser grandes motores multidisciplinares, una universidad puede contratar sus servicios pensando en grupos de investigación variados).

El ejemplo de ProQuest LLC ayuda a entender el funcionamiento de tales empresas. Este gigante de la información, aunque posee un portal de búsqueda principal (http://www.proquest.umi.com), ofrece otros productos diferentes, como es el caso de su portal Cambridge Scientific Abstracts (www.csa.com), muy útil en educación musical ya que incluye la base de datos del Educational Resources Information Center. Creado por el U.S. Department of Education y gestionado por CSA, el ERIC da acceso a más de 1,3 millones de entradas bibliográficas sobre educación, incluyendo artículos de revistas, libros, tesis, informes legislativos, comunicaciones en conferencias. El encontrar información distinta en dos portales que son de una misma compañía confirma que ningún portal puede ser tomado como la referencia absoluta en nuestra área.

Pero centrémonos en el principal motor de búsqueda de ProQuest. En cuanto a la investigación en educación musical, las bases más importantes que proporciona son: Arts and Humanities Full Text -la cual contiene el catálogo completo del RILM-, Dissertation and Theses A\&I-que cuenta con el mayor repertorio de tesis y tesinas del mundo- y Proquest Psychology Journals -que posee una gran cantidad de artículos de revistas a texto completo. Como muestra de sus enormes posibilidades se puede realizar una búsqueda relativamente acotada: al introducir los descriptores "music education" + "teacher education", en referencia a la formación de los profesores de música, aparecen 390 entradas. En la Figura 2, que muestra los dos primeros resultados por orden de relevancia, se observa que la información ofrecida no difiere mucho de, por ejemplo, la base de datos ISOC. 


\section{ProQuest}

390 documentos encontrados para: (music education) AND (teacher education)

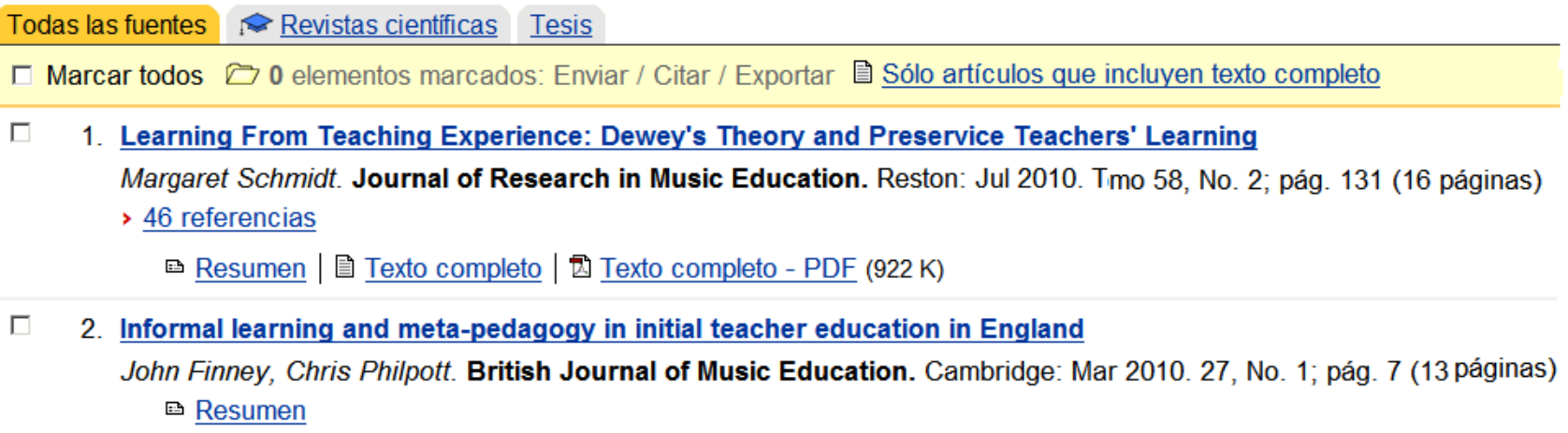

Fig. 2

Ahora bien, en la primera entrada se observa un enlace a las citas del documento. En este caso, se trata de un artículo del Journal of Research in Music Education, publicación que indexa todas las referencias bibliográficas que aparecen en sus textos, lo que supone un avance importantísimo en el manejo de grandes cantidades de información ya que, a su vez, en cada referencia aparece quién ha citado el mismo artículo (Fig. 3). Esta bibliografía relacional es posible gracias al DOI.

\section{Referencias \\ bibliográficas}

\section{ProQuest}

1. Influences on Instrumental Music Teaching

Bauer, William I., Berg, Margaret H. Bulletin of the Council for Research in Music Education [H.W. Wilson - EDUC] 2001. pág. 53

$>$ Citado por 4

2. The nature of expertise in teaching

D. C. Berliner. 2008/1992. In Handbook of Research on Teacher Education , K. E. Demers (ed.). New York, NY: Routledge pág. 808-823

$>$ Citado por 2

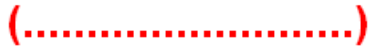

46. Are the Effects of University Teacher Education "Washed Out" by School Experience?

Kenneth M Zeichner, Kenneth M Zeichner, B Robert Tabachnick. Journal of Teacher Education. May 1981. Tomo 32, No.

7-11

$>$ Citado por 55

Fig. 3

\section{CRIBA DE DOCUMENTOS Y VALIDEZ DE LAS TÉCNICAS BIBLIOMÉTRICAS EN EL CAMPO DE LA EDUCACIÓN MUSICAL}

Como hemos visto anteriormente, el corpus científico en educación musical crece a un ritmo exponencial y no existe una única herramienta para gestionarlo. Este exceso de información supone un grave peligro para la tarea investigadora -"el cerebro no puede funcionar de forma eficaz con demasiada información" (SMALL y VORGAN, 2009:22). En este sentido, podemos afirmar que una investigación eficiente depende más de nuestra capacidad para seleccionar las fuentes más idóneas que de nuestro volumen de trabajo. Pero ¿cómo seleccionar los textos más relevantes? ¿Qué documentos siguen teniendo validez hoy día? ¿Quiénes son los autores más "autorizados"?

Ya en 1992, CRABTREE y FOSTER son conscientes de la dificultad de establecer una bibliografía de referencia en investigación musical dada la enorme cantidad de textos científicos que aparecen a partir de la década de 1980. Tanto es así que su sourcebook (libro de fuentes bibliográficas) muestra una tendencia generalizada en muchos trabajos de investigación: la citación de autores que se encuentran dentro de círculos académicos próximos. PRICE y sus colaboradores (2010) confirmaron esta realidad a partir del estudio bibliométrico de las comunicaciones presentadas en los seminarios 
de la Comisión de Investigación de la International Society for Music Education (ISME), durante el periodo 1988-2006:

“[...] mediante el acceso electrónico es posible consultar revistas, libros y otros documentos académicos de entre una miríada de fuentes presentes en diversas publicaciones internacionales. Sin embargo, se podría afirmar que hay un fuerte arraigo geográfico en cuanto a fuentes en todos los documentos de investigación" (PRICE et al., 2010:92).

Según los autores, una de las razones de este arraigo es la idiosincrasia del sistema educativo y del tipo música sobre el que cada investigador trabaja, por ejemplo, si trabajamos en la didáctica del flamenco, será inevitable acotar las citas al ámbito español. Con todo y con eso, muchos autores se limitan a una bibliografía de entorno próximo bien porque no conocen las nuevas tecnologías aquí tratadas, bien porque no saben realizar una criba eficiente de los textos.

\section{Nuevas herramientas de gestión bibliográfica}

Frente a este panorama, la biblioteconomía ha desarrollado herramientas que pueden ayudar a organizar una bibliografía precisa y de calidad para una problemática concreta. Una de los avances más importantes ha sido la bibliografía relacional -ya mencionada en el punto anterior. En los últimos años han aparecido nuevas aplicaciones que suponen otra vuelta de tuerca a esta tecnología, siendo la Web of Knowledge su principal impulsora (Fig. 4).

\section{ISI Web of Knowledge}

\section{THOMSON REUTERS}

Title: Observation and analysis of Suzuki string teaching Author(s): Colprit EJ

Source: JOURNAL OF RESEARCH IN MUSIC EDUCATION Volume: 48 Issue: 3 Pages: 206-221 Published: FAL 2000 Times Cited: 13

Fig. 4.

Así, ante un artículo cualquiera (Fig. 4), la web ofrece (Fig. 5) los artículos que citan a este documento - un total de trece-, los artículos que aparecen en su bibliografía -references: 45- y los artículos relacionados temáticamente-related records.

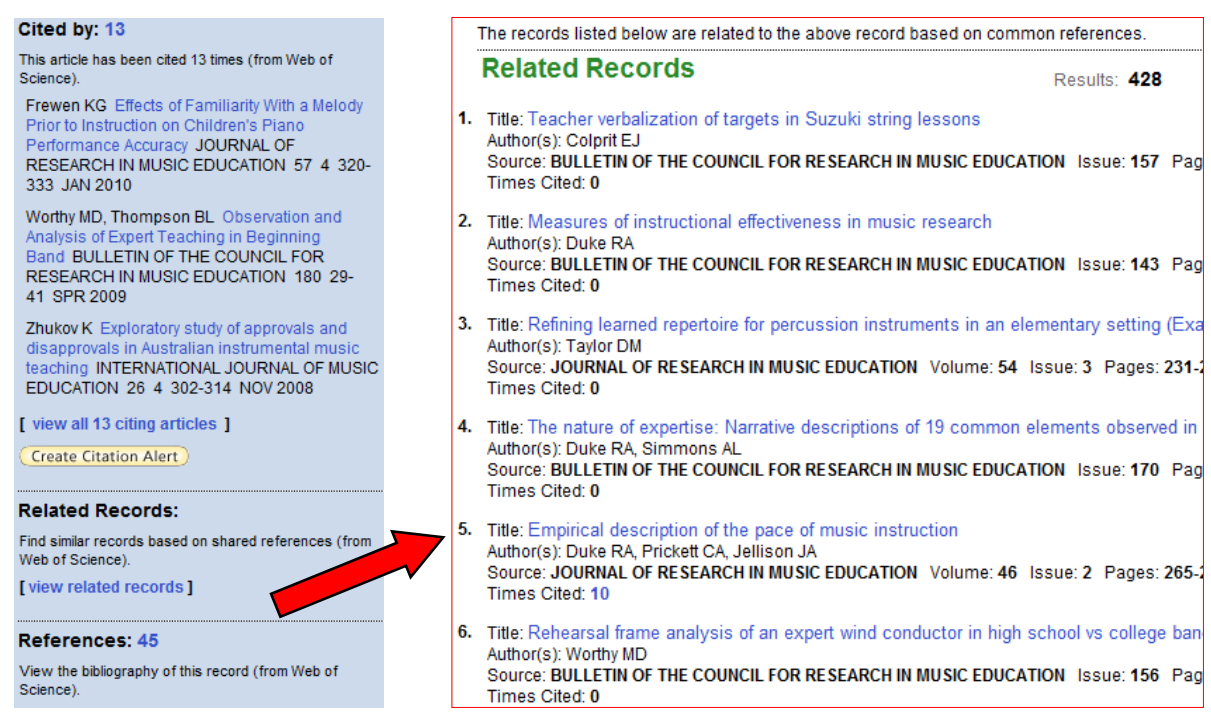

Fig. 5

Sin duda, esta última herramienta (related records) supone un avance significativo, ya que artículos que en un principio no guardan ninguna similitud en cuanto al título, están altamente relacionados mediante sus descriptores. 
La última aplicación desarrollada por la WOK son los mapas de citación, en los que se crea un diagrama con etiquetas de cada uno de los documentos de la bibliografía. Estas etiquetas se organizan mediante colores, de ahí que, si queremos diferenciar los artículos que pertenecen a una revista concreta del resto, un color en particular determinará todos los artículos de una misma revista; pudiendo hacer lo propio con los autores del texto (un color por autor), año de publicación (un color para cada año), tipo de documento, país, lengua del documento, etc. Si creamos el mapa de citación del ejemplo anterior y lo organizamos mediante revistas de pertenencia (Fig. 6), el color verde, por ejemplo, marcará todos los artículos que han sido publicados en el Bulletin of the Council for Research in Music Education.

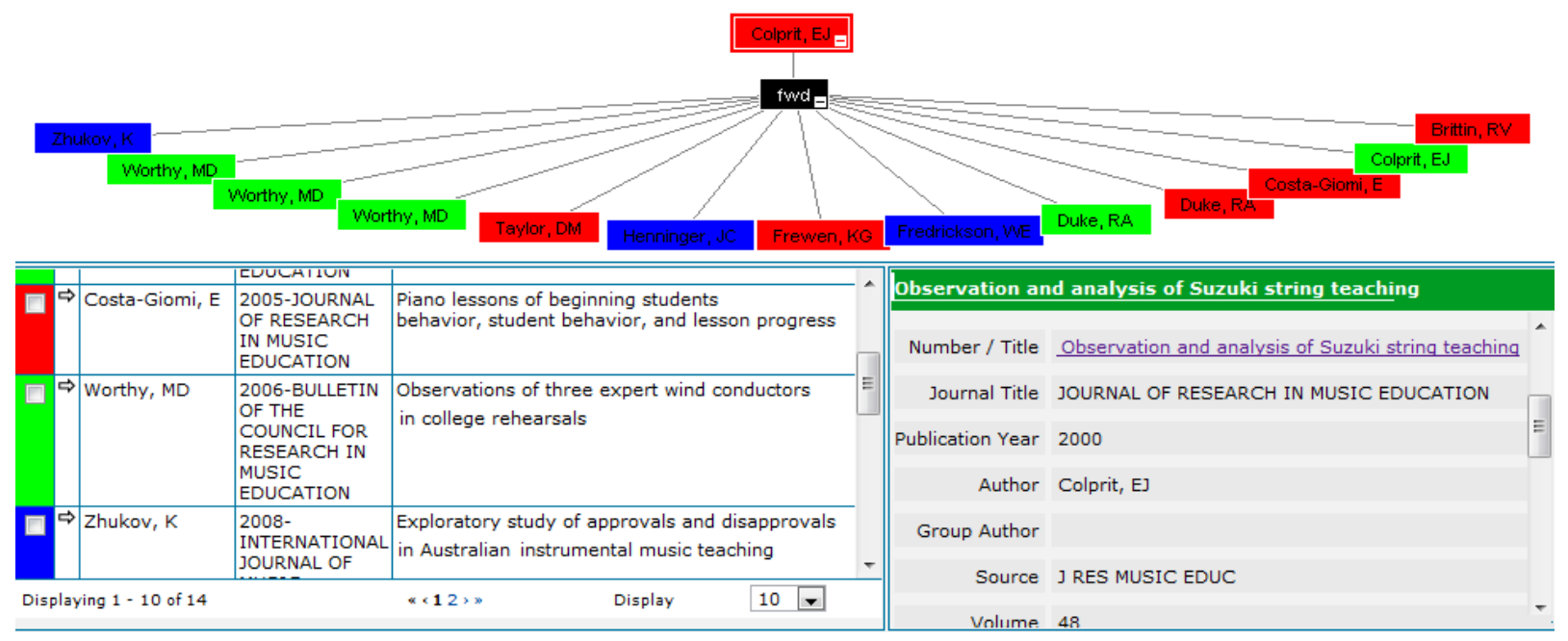

Fig. 6

Esta aplicación puede parecer no demasiado útil en nuestro campo dada la baja tasa de citación de ciertos artículos. No obstante, la parcela de la investigación en educación musical directamente relacionada con la psicología cognitiva genera trabajos que tienen un impacto enorme -citados por más de cincuenta artículos- y requieren, por tanto, de herramientas más complejas de análisis.

\section{Técnicas bibliométricas e investigación musical}

A pesar de la utilidad de las técnicas anteriormente descritas, la comunidad científica ha visto la necesidad de evaluar la bibliografía científica mediante lo que ha venido a llamarse bibliometría, es decir, el estudio del comportamiento estadístico de los diferentes elementos que conforman un área del saber. Conviene recalcar que los estudios bibliométricos son limitados, sobre todo en lo relativo a los indicadores de visibilidad o impacto. En primer lugar, porque no existe una única base de datos que recoja toda la producción científica; en segundo lugar, porque, como subraya la documentalista María Ángeles Zulueta (2002:135), falta un criterio universalmente aceptado acerca del sentido de las citas, ya que los autores no siguen siempre comportamientos lógicos o consistentes en sus hábitos de citación. Además, hay que interrogarse sobre la validez de este tipo de técnicas en un área como la nuestra, en la cual confluyen disciplinas diversas (musicología, psicología, didáctica, sociología, semiología, etc.).

Según GiMÉNEZ, GóMEZ y VÁZQuEZ (2001:41), el método de selección basado en el factor de impacto, por ejemplo, origina que prevalezcan la lengua inglesa frente a otras, las comunidades grandes frente a las pequeñas o los temas de investigación de evolución rápida frente a otros más estables: "será adecuado para las ciencias experimentales, pero lo será menos para las aplicadas o las Ciencias Sociales y prácticamente es inaplicable en el caso de las Humanidades”. Por tanto ¿qué pautas de citación guían los trabajos de investigación en educación musical? Si bien esta pregunta daría para varias tesis doctorales, sí que se pueden establecer algunas reflexiones al respecto. 
Primero, aunque la investigación en educación musical contenga elementos de las Humanidades - por la naturaleza artística de su savoir savant o saber de base (CHEVALLARD, 1985)- su núcleo principal pertenece a las Ciencias Sociales, por lo que sus patrones de citación se igualan a los de éstas o incluso, en ocasiones puntuales, pueden llegar a parecerse a los de las Ciencias Experimentales. En consecuencia, el modelo bibliográfico en nuestro campo difiere enormemente de aquel de la musicología aplicada e histórica. DiODATO y SMITH (1993:110) corroboraron esta realidad mediante el estudio de la edad media de citación y la obsolescencia de 9.850 artículos en educación musical, teoría musical y musicología aplicada e histórica, llegando a la conclusión de que tanto "la educación musical como la teoría musical muestran patrones de obsolescencia próximos a aquellos encontrados en las ciencias y la tecnología", que en el caso de nuestra área corresponde a una vida media de citación de entre diez y quince años.

Segundo, las investigaciones sobre el proceso de enseñanza-aprendizaje de la música tienen un interés y una validez internacionales, existiendo un corpus unificado de bibliografía científica. Aunque la problemática tratada en cualquier artículo de este campo sea de carácter local, sus conclusiones pueden ser extrapoladas a otros contextos o servir como base de reflexión para otras realidades docentes. En este sentido hay señalar la importancia del inglés como lengua vehicular, sobre todo en publicaciones de comunidades científicas pequeñas, si éstas quieren tener un impacto a nivel internacional.

Si, como acabamos de ver, la investigación en educación musical se aproxima a los parámetros bibliográficos de las Ciencias Sociales, bien es cierto que los indicadores de visibilidad por autores presentan algunos problemas. El principal inconveniente es que, al no existir una única base de datos que reúna el grueso de la bibliografía en nuestra área, los trabajos de un autor concreto pueden estar diseminados entre varios motores de búsqueda. El resultado: muy pocos investigadores son susceptibles de un análisis de impacto. En cuanto a aquellos pocos que sí generan suficientes textos como para someterse a estas técnicas hay que decir que son, en su mayoría, anglosajones (por ejemplo, David J. Hargreaves, Clifford Madsen, Susan J. Hallam o Martin J. Bergee).

El caso de los autores latinoamericanos también es parecido -o por lo menos de aquellos que sólo escriben en castellano. Su baja tasa de citación hace que el índice de impacto no resulte demasiado importante. De hecho, en el Índice de Impacto de Revistas Españolas de Ciencias Sociales (IN-RECS) no aparecen las estadísticas pormenorizadas de los autores más citados en las publicaciones analizadas (Lorenzo Quiles, Oriol de Alarcón, Bermell Corral o Alsina i Masmitja), como sí ocurre con los investigadores de otras áreas con mayor alcance, como la didáctica de las ciencias, por ejemplo.

\section{El factor de impacto de las revistas}

Si bien existen actualmente varios "criterios de calidad" para evaluar revistas científicas, creemos que es pertinente centrarnos en el factor de impacto, porque es el que, de forma más sencilla, nos sirve para comparar nuestra bibliografía con el resto de las ciencias sociales. Dado que el factor de impacto individual no tiene mucho valor en investigación en educación musical, debemos preguntarnos si los índices de visibilidad de las revistas en este campo son de más utilidad o no. Existe una infinidad de aplicaciones que evalúan la calidad de las revistas y su factor de impacto, las más importantes para nosotros son: Social Science Citation Index (accesible a través de la WOK), Journal Citation Reports (desarrollado por Thomson Reuters), SCImago Journal and Country Rank (que pretende ser una alternativa al JCR), IN-RECS y Revistas Españolas de Ciencias Sociales y Humanas (RESH).

El funcionamiento de todos estos índices es parecido, aunque los criterios de evaluación difieran levemente. Todos ellos toman bases de datos como referencia, es decir, evalúan la actividad científica partir de los documentos contenidos en éstas. Por tanto, los índices no trabajan sobre el conjunto de las revistas de un área de conocimiento sino sobre aquellas que aparecen en las bases de 
datos de referencia (SSCI y JCR sobre Thomson ISI, SJR sobre Scopus, IN-RECS sobre Latindex, Cindoc y Rebiun, entre otras). En consecuencia, los índices evalúan revistas que ya han pasado una criba de calidad para formar parte de una base de datos concreta, es decir, todas son publicaciones consolidadas. La pregunta es si existen o no diferencias notables de calidad entre aquellas revistas en educación musical que ya están indexadas.

La aplicación Journal Analyzer de Scopus puede ayudarnos a salir de dudas. Al realizar un análisis comparado de las cuatro revistas en educación musical que indexa, obtenemos diferentes herramientas de evaluación de la calidad. Si tomamos el índice Citations -que indica el total de citas recibidas por una revista en cada año- (Fig. 7), podemos ver que el Journal of Research in Music Education es la publicación que más citas genera; podríamos pensar, por tanto, que su factor de impacto es mayor que el del resto de revistas.

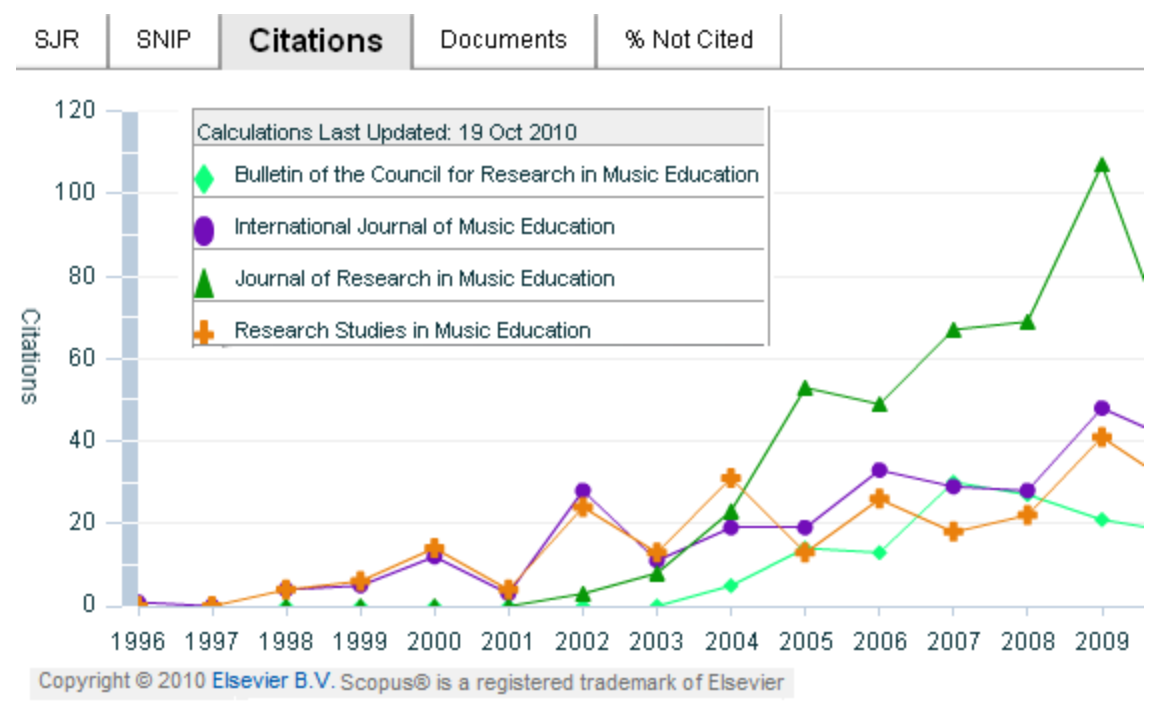

Fig. 7

Ahora bien, si aplicamos el SCImago Journal and Country Rank -SJR-(Imagen 8), que mide la cantidad media de citas que genera un artículo y la importancia de cada una de ellas, podemos observar que no hay apenas diferencia entre unas publicaciones y otras.

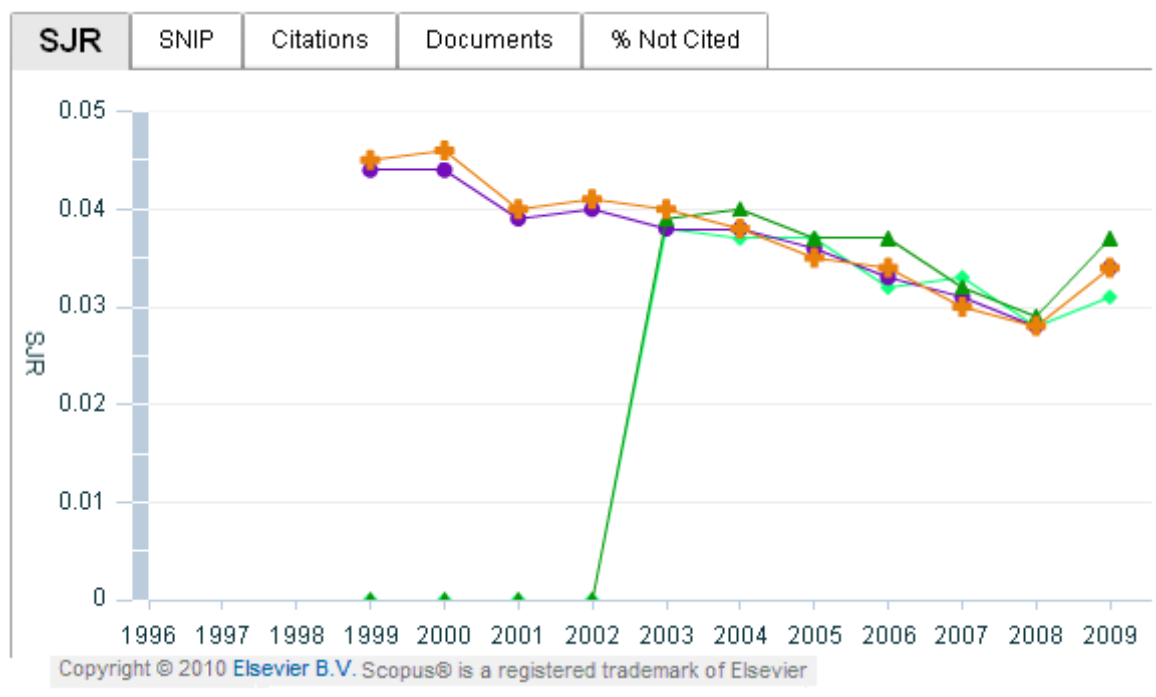

Fig. 8

De esta manera, se puede afirmar que los índices de impacto no son realmente útiles para las revistas en educación musical, ya que la verdadera referencia en cuanto a calidad es que la revista 
esté indexada en una base de datos de renombre. Si hablamos de los índices para las revistas españolas, llegaremos prácticamente a las mismas conclusiones. Sin embargo, aunque estos índices no sean muy prácticos para comparar revistas en educación musical entre sí, sí que lo son para establecer la salud de nuestra bibliografía científica respecto de las Ciencias Sociales en general. Pongamos como ejemplo las revistas españolas.

El índice de citas del RESH (Revistas Españolas de Ciencias Sociales y Humanas), contiene, en su área de educación, las revistas Música y Educación y Eufonía. Como se puede observar en el índice de citas del periodo 1998-2003 (Fig. 9), estas dos publicaciones aparecen en los puestos 32 y 34 , respectivamente, de un total de 77 revistas analizadas, es decir, muestran índices de impacto muy parecidos. Hay que señalar que los datos de este índice se aproximan mucho a aquellos de las revistas anglófonas en educación musical analizadas en la Fig. 8, entre 0,03 y 0,04 puntos, lo que sitúa a nuestra área de conocimiento dentro de la media de los índices de visibilidad de las ciencias de la educación.

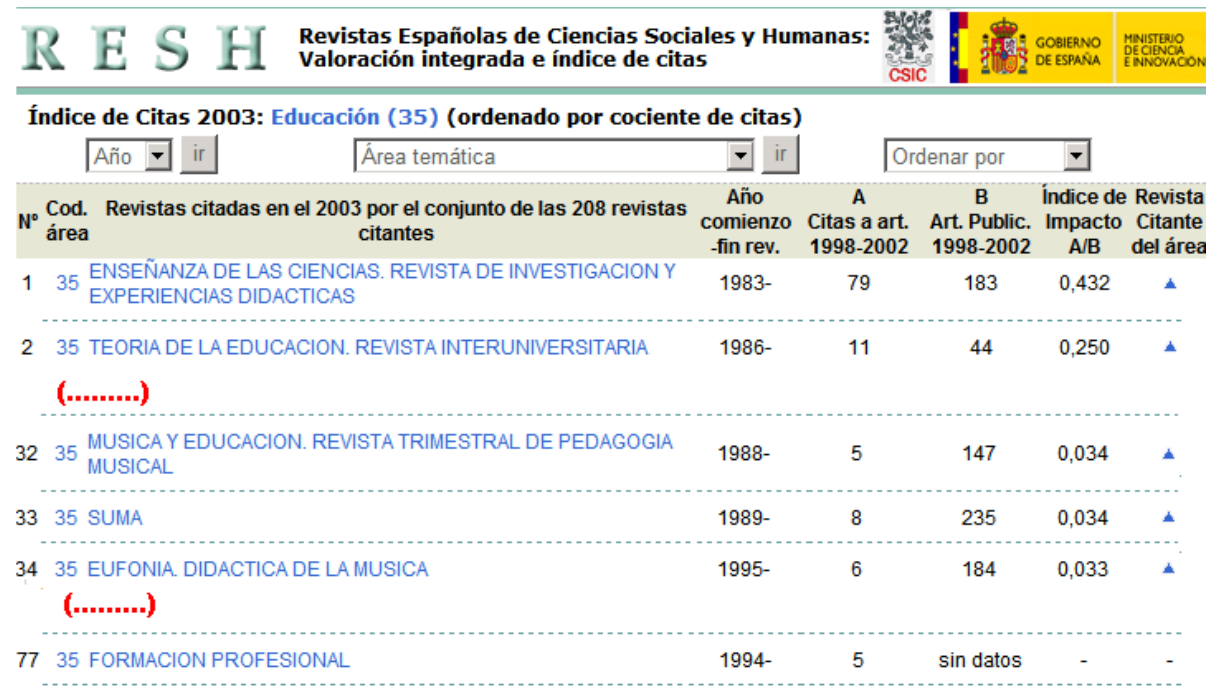

Fig. 9

Sin embargo, RESH únicamente contiene dos revistas en educación musical y ambas son publicaciones en papel. ¿Qué pasaría, por tanto, si añadiésemos a estos índices las revistas digitales? El Índice de Impacto de Revistas Españolas de Ciencias Sociales (IN-RECS) recoge un total de 156 revistas en su apartado Educación. Además de las citadas Música y Educación y Eufonía, indexa las dos revistas digitales más importantes en educación musical en castellano: Revista Electrónica Complutense en Investigación Musical en Musical y Revista Electrónica de LEEME (Lista Electrónica Europea de Música en la Educación). Como se puede observar en el impacto acumulativo del periodo 1994-2009 (Fig. 10), todas las revistas aumentan su índice de visibilidad y las dos publicaciones de educación musical en papel mejoran sus resultados respecto del total del área de Educación. Esto es debido, evidentemente, a que las revistas digitales aumentan el conjunto de citas con origen en revistas en papel. Otro dato a destacar es que la Revista Electrónica Complutense en Investigación en Educación Musical obtiene excelentes resultados a pesar de su juventud, llegando incluso a conseguir 2 citas internacionales con tan solo 18 artículos publicados. Esto corrobora la idea de que las revistas electrónicas son un canal eficiente para la difusión del conocimiento científico en el siglo XXI. 


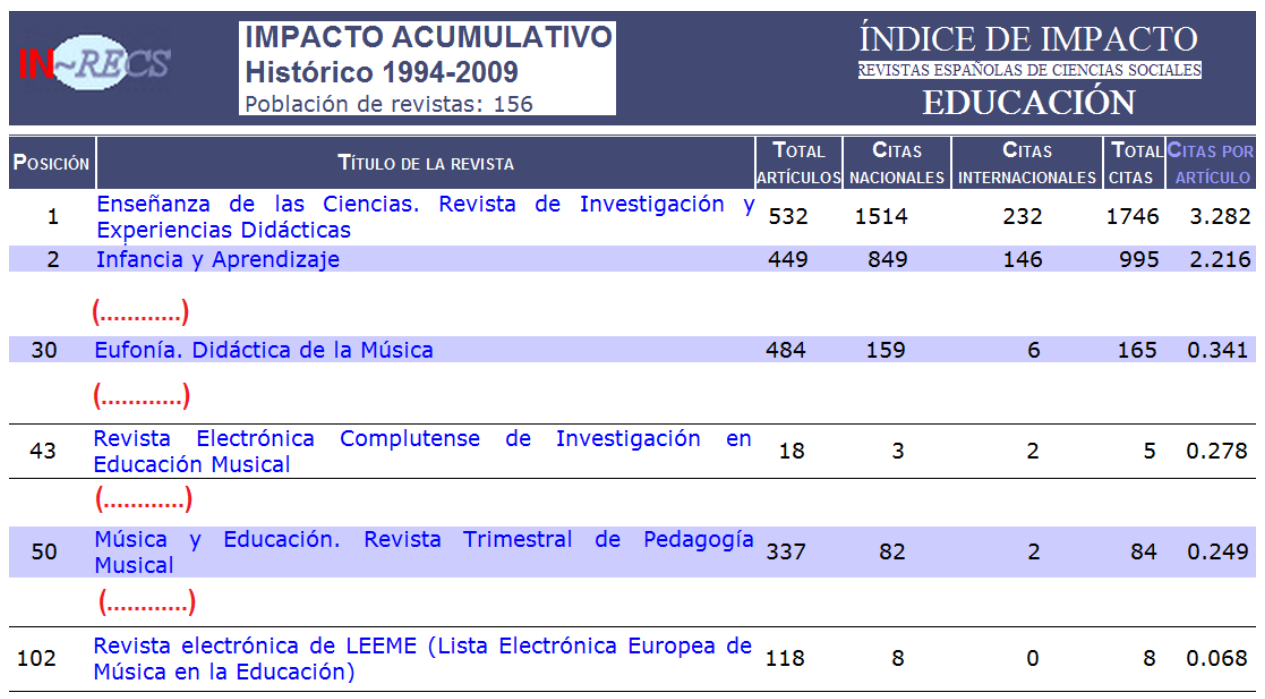

Fig. 10

\section{CONCLUSIONES}

La síntesis que acabamos de realizar pretende ser una llamada de atención a toda la comunidad que investiga en educación musical sobre la velocidad de los cambios que se han producido en materia de documentación en los últimos años. El texto digital e internet posibilitan el crecimiento exponencial de textos científicos en nuestro campo, lo que genera la necesidad de una actualización continua en cuanto a técnicas documentales. Los investigadores iberoamericanos debemos aprovechar este hecho para salvar unos metros en la larga distancia que nos separa de la investigación anglosajona (muy condicionada por la gran cantidad de medios bibliográficos que han tenido tradicionalmente a su alcance).

Debemos tener en cuenta que las publicaciones digitales nutren y consolidan la bibliografía en nuestra área y no suponen un descenso en la calidad de la misma. Además, este tipo de documentos ha permitido la creación de un corpus internacional, más o menos unificado, en investigación en educación musical. No sabemos con certeza si las revistas en papel desaparecerán en un futuro, lo que sí podemos dar por seguro es que las publicaciones digitales pronto desbancarán en importancia y uso a aquellas físicas. Si se llegase además a generalizar la gratuidad de los contenidos, esto supondría otro avance importante para las universidades europeas -las cuales manejan presupuestos menores que las grandes universidades americanas.

En cuanto a la aplicación de técnicas bibliométricas, queremos recalcar que, hoy en día, la verdadera prueba de calidad se produce a nivel de la indexación de las revistas en las bases de datos, lo que hace que los índices de impacto sólo sean útiles para medir la salud de nuestra bibliografía científica respecto del conjunto de las ciencias sociales. Por su parte, los rankings de autores o trabajos más citados son inaplicables en la investigación en educación musical, sólo unos pocos autores anglófonos son susceptibles de este análisis.

En definitiva, actualmente, todo trabajo sobre educación musical -por local que sea la problemática tratada- debe partir de una fundamentación teórica de carácter internacional. Esta primera fase requiere del manejo de las técnicas de búsqueda documental aquí descritas. Por tanto, existe la necesidad de una alfabetización informacional (JOHNSTON y WeBER, 2007) en la formación de los investigadores en nuestra área. Este proceso consiste en el desarrollo de habilidades ligadas al proceso de búsqueda, selección y recuperación de documentos pertinentes para nuestra investigación, es decir, el alfa de todo proceso científico. 


\section{Referencias}

BACCINO, T. (2004) : La lecture électronique, Grenoble, Presses Universitaires de Grenoble.

Chevallard, Y. (1985): La transposition didactique: Du savoir savant au savoir enseigné. Paris, La Pensée Sauvage.

Crabtree, P. D.; Foster, D. H. (1993): Sourcebook for research in music. Bloomington, Indiana University Press.

Diodato, V.; Smith, F. (1993): "Obsolescence of music literature". Journal of the American Society for Information Science, 44(2), 101-112.

FRANCES, R. (1958) : La perception de la musique. Paris, Vrin.

GIMÉNEZ E.; GóMEZ I.; VÁZQUEZ M. (2001): "Difusión nacional e internacional de las revistas científicas”. En A. RomÁn (ed.): Guía de buenos usos. La edición de revistas científicas (págs. 35- 46). Madrid, Consejo Superior de Investigaciones Científicas.

GimÉNEZ Toledo, E. (2006): “Revistas electrónicas”. En M. MARTíNEZ y A. RodRíGuez (coords.): La información especializada en internet (págs. 117-138). Madrid, Consejo Superior de Investigaciones Científicas.

IMBERTY, M. (1969): L'acquisition des structures tonales chez l'enfant. Paris, Klincksieck.

Johnston, B.; WeBber, S. (2007): "Cómo podríamos pensar: Alfabetización informacional como una disciplina en la era de la información”. Anales de Documentación, 10, 491-504.

Lillo Hidalgo, V. (2009): "Publicar una revista científica en Internet: ¿Cómo hacerlo?”. Unelibros, 19, 8. Consultado en http://www.une.es/Ent/Items/ItemDetail.aspx?ID=2885.

Osca Lluch; Haba, J.; Navarro G.; Minguez, O.; Velasco, E.; Salom, L. (2008): "Difusión y factor de impacto nacional e internacional de las revistas científicas españolas". Anales de documentación, 11, 145-164.

Price, H. E. Madsen, C. K.; Cornacchio, R.; WebB, M. (2010): "Examination of the international scope of papers presented at the International Society for Music Education Research Comissions Seminars". Journal Research in Music Education, 58(1), 90-96.

Sloboda, J. A. (1985): The musical mind: The cognitive psychology of music. Oxford, Oxford University Press.

Small, G.; Vorgan, G. (2009): El cerebro digital. Cómo las nuevas tecnologías están cambiando nuestra mente. Barcelona, Urano.

Zulueta, M. A. (2002): "Bibliometría y métodos bibliométricos”. En J. LóPEZ YePes: Manual de Ciencias de la Documentación (págs. 117-136). Madrid, Pirámide. 